Trends Biochem Sci. 2013 March ; 38(3): 121-130. doi:10.1016/j.tibs.2012.11.008.

\title{
Secreted protein kinases
}

\author{
Vincent S. Tagliabracci ${ }^{1}$, Lorenzo A. Pinna ${ }^{2}$, and Jack E. Dixon ${ }^{1,3,4,{ }^{*}}$ \\ ${ }^{1}$ Department of Pharmacology, University of California, San Diego, La Jolla CA \\ ${ }^{2}$ Department of Biomedical Sciences, University of Padova and CNR Institute of Neurosciences, \\ Padova Italy \\ ${ }^{3}$ Howard Hughes Medical Institute, University of California, San Diego, La Jolla CA \\ ${ }^{4}$ Department of Chemistry and Biochemistry and Department of Cellular and Molecular Medicine, \\ University of California, San Diego, La Jolla CA
}

\begin{abstract}
Protein kinases constitute one of the largest gene families and they control many aspects of cellular life. In retrospect, the first indication for their existence was reported 130 years ago when the secreted protein, casein, was shown to contain phosphate. Despite its identification as the first phosphoprotein, the responsible kinase has remained obscure. This conundrum was solved with the discovery of a novel family of atypical protein kinases that are secreted and appear to phosphorylate numerous extracellular proteins, including casein. Fam20C, the archetypical member, phosphorylates secreted proteins within Ser-x-Glu/pSer motifs. This discovery has solved a 130-year-old mystery and has shed light on several human disorders of biomineralization.
\end{abstract}

\section{The casein paradox}

The first phosphoprotein was reported in 1883 by Olof Hammarsten when he detected phosphorous in the secreted protein casein (Glossary) [1]. In hindsight this was the first indication for the existence of protein kinases. Ironically, it would take nearly 130 years for the molecular identification of the kinase that phosphorylates casein [2]. In the meantime, the use of casein as a model substrate in protein phosphorylation research was flourishing and contributed significantly to what we know today about protein kinases (Table 1).

Hammarsten detected stoichiometric amounts of phosphate in the casein he had prepared; however, covalently bound protein phosphate was not detected until the early 1930's when Fritz Lipmann and Phoebus Levene demonstrated that casein and the secreted protein egg yolk phosvitin contained phosphoserine [3, 4]. Some 70 years after Hammarsten's discovery, Burnett and Kennedy detected a novel activity from rat liver mitochondrial extracts that was capable of transferring the terminal phosphate of ATP onto Ser residues within casein [5]. This was the first detection of a protein kinase activity. Soon afterwards, Krebs and Fischer found that the interconversion of phosphorylase $b$ to phosphorylase $a$ (the enzyme that catalyses the rate-limiting step of glycogenolysis) involved a phosphorylation/ dephosphorylation mechanism [6]. A few years later, Rabinowitz and Lipmann demonstrated that casein kinase activity, which could also phosphorylate phosvitin, (Box 1)

() 2012 Elsevier Ltd. All rights reserved.

*Correspondence to: jedixon@ucsd.edu.

Publisher's Disclaimer: This is a PDF file of an unedited manuscript that has been accepted for publication. As a service to our customers we are providing this early version of the manuscript. The manuscript will undergo copyediting, typesetting, and review of the resulting proof before it is published in its final citable form. Please note that during the production process errors may be discovered which could affect the content, and all legal disclaimers that apply to the journal pertain. 
was present in brain and yeast and was distinct from the phosphorylase kinase described by Krebs and Fischer [7].

\section{Box 1}

\section{The genuine "phosvitin kinase": a persisting mystery}

In the infancy of studies on protein kinases, egg yolk phosvitin (previously known as "vitellinic acid") was also widely used, in addition to casein, as an excellent artificial substrate. Therefore, protein kinases that could phosphorylate casein and phosvitin were often referred to as "phosvitin kinases". For decades, phosvitin was the only other known phosphoprotein available in large amounts and it was the substrate Rabinowitz and Lipmann used to show that the protein kinase activity detected by Burnett and Kennedy was different from the only protein kinase known at that time, phosphorylase kinase [6, 7]. Later, phosvitin was used to demonstrate that phosphoserine can replace carboxylic side chains as specificity determinants [72]. Phosvitin is composed of long stretches of up to 14 entirely phosphorylated serines, which in a few instances are located adjacent to the $\mathrm{C}$ terminus of a non-phosphorylated threonine. Unlike casein, in which the triplets of phosphoserines are adjacent to Glu-Glu doublets, phosvitin lacks this motif and cannot sustain non-primed phosphorylation by both CK2 and G-CK. Indeed, phosvitin is almost entirely lacking in acidic residues; apart from its huge content of phosphate, it is a fairly basic protein in its unphosphorylated form. In conclusion, none of the acidophilic kinases known to date can be responsible for the initiation of phosvitin phosphorylation based on their specificity determinants. The discovery that Fam20C is the same entity as G-CK has solved the "very cold case" of casein dating back to 1883, but not that of phosvitin, dating back to 1900 [73].

Over the next 10 years the "casein kinases" were characterized and shown to be distinct from the metabolic or second messenger dependent protein kinases involved in glycogen metabolism $[6,8]$. These studies, along with many others, established the existence of two distinct, ubiquitous protein kinases termed casein kinase- 1 and casein kinase-2, named because they were capable of phosphorylating casein in vitro $[9,10]$. These enzymes are mainly nuclear and cytosolic and would be highly unlikely to encounter casein in the secretory pathway. As a result, they were later renamed protein kinase CK1 and protein kinase CK2 to avoid confusion with the genuine casein kinase(s) responsible for phosphorylating casein in vivo [11]. Genuine casein kinase(s) were less well understood and most studies utilized extracts of mammary gland particulate fractions to investigate their properties.

One such kinase was biochemically characterized and named Golgi enriched fraction casein kinase (GEF-CK, later shortened into G-CK). Its activity was initially detected in lactating mammary glands, from which it was further characterized and partially purified as an $\sim 70$ $\mathrm{kDa}$ enzyme $[12,13]$. However, it was never purified to homogeneity or identified molecularly. Scrutinizing the sequences of casein fractions revealed the presence of several phosphoserines that were often clustered in triplets and invariably displayed an S-x-E/pS motif $[14,15]$. These sites are similar but not identical to those recognized by CK1 (pS-x-x$\mathrm{S} / \mathrm{T}$ ) and CK2 (S/T-x-x-E/D/pS). An important advancement in the studies of G-CK was the development of a synthetic peptide substrate corresponding to a phosphorylation site in bovine $\beta$-casein, $\beta$ (28-40). This peptide could be phosphorylated by G-CK but was an extremely poor substrate for CK1 and CK2 [16-18]. Therefore, this peptide was used to assay G-CK and subsequently used to follow its partial purification from many tissues [19, 20]. The G-CK also has unusual biochemical properties for a protein kinase such as insensitivity to staurosporine, a protein kinase inhibitor [19]. The G-CK prefers $\mathrm{Mn}^{2+}$ (and 
to a lesser extent $\mathrm{Co}^{2+}$ ) over $\mathrm{Mg}^{2+}$ and GTP cannot substitute ATP as the phosphate donor [19]. In addition to casein, subsequent studies identified a number of secreted proteins that were substrates for the G-CK, further necessitating the need to determine the primary amino acid sequence of this elusive protein kinase.

\section{The real casein kinase}

Virtually all proteins that enter the secretory pathway, including the caseins, have a short sequence of hydrophobic amino acids at the $\mathrm{N}$-terminus commonly referred to as the signal peptide (SP). Numerous secreted proteins are phosphorylated by unidentified protein kinases present within the lumen of the Golgi apparatus and/or in the extracellular space (Box 2). However, these enzymes are poorly characterized and their activities have been ascribed to the category of orphan enzymes (enzymes that have not been molecularly identified [21]). It seemed likely that the kinase(s) responsible for phosphorylating secreted proteins would be present within the secretory pathway and therefore would contain a SP. Therefore, to identify possible candidates for the G-CK, the known protein kinases were searched for those that contained an $\mathrm{N}$-terminal SP, a C-terminal kinase like domain and no predicted transmembrane helix [2](Figure 1A). This structural design would orient the kinase like domain in the lumen of the ER/Golgi, in close proximity to proteins destined for secretion. Four-jointed (fj) is one such kinase that localizes to the Golgi in Drosophila and phosphorylates the extracellular domain of atypical cadherins during transit through the secretory pathway [22]. Using the human four-jointed (fjx1) amino acid sequence, a family of related proteins was identified by PSI-BLAST (Figure 1B). These proteins are distantly related to the mammalian lipid kinase phosphatidylinositol 4-kinase-II (PI4KII) and the bacterial kinase HipA and are predicted to have a SP [23, 24]. The C-terminal domain shows only weak sequence similarity to canonical protein kinases yet several conserved residues essential for protein kinase activity are present. The identified family includes members of the family with sequence similarity $\underline{20}$ (Fam20) include Fam20A, Fam20B and Fam20C and have been genetically implicated in mineralized tissue homeostasis. Also in the family of fjx1-related proteins are members of the family with sequence similarity 198 (Fam198), which include Fam198A and Fam198B and have yet to be functionally characterized. These proteins were expressed in mammalian cells, immunopurified, then assayed to phosphorylate casein. Fam20C phosphorylated casein and possessed all the biochemical properties of the G-CK including Golgi localization, ability to phosphorylate the casein peptide $\beta(28-40)$, preference for $\mathrm{Mn}^{2+}$ and $\mathrm{Co}^{2+}$ over $\mathrm{Mg}^{2+}$, and extreme insensitivity to staurosporine $[2,25]$ Fam20C co-elutes with casein kinase activity when bovine whey is fractionated by Q-Sepharose and Mono Q chromatography [2, 20]. Furthermore, Fam20C is present in highly purified preparations of G-CK from lactating mammary gland, thus providing incontrovertible evidence that G-CK and Fam20C are one and the same [26].

\section{Box 2}

\section{Where is the phosphorylation occuring?}

The phosphorylation of secreted proteins can occur in the lumen of the Golgi and/or extracellularly [74] [75] (Figure I). The rate of phosphate transfer will depend not only on the local concentrations of kinase and protein substrate but also on the amount of ATP. The Fam $20 \mathrm{C} \mathrm{K}_{\mathrm{m}}$ for ATP is $\sim 78 \mu \mathrm{M}$ as reported by Irvine and colleagues [25]. Indeed, extracellular ATP concentrations above $100 \mu \mathrm{M}$ have been documented, suggesting that Fam20C may function extracellularly [75]. Although this awaits confirmation, it is clear that Fam20C catalyzes phosphorylation within the cell because coexpression of Fam20C with OPN results in the phosphorylation of both intracellular and secreted OPN [2]. Intracellular phosphorylation of secreted proteins requires ATP to be accessible to protein kinases present in the Golgi lumen and therefore must cross the 
Golgi membrane. The transporters that facilitate ATP entry from the cytosol into the lumen of the Golgi are poorly understood. The Golgi membrane ATP transporter was purified 70,000-fold from crude rat liver Golgi membranes but the molecular identity of the transporter was never determined [76]. The Golgi pH Regulator (GPHR) was identified by Maeda et al. to be a novel anion channel that is essential for the acidification and functions of the Golgi [77]. This channel showed a striking resemblance to two anion channels that were shown to mediate ATP transport into the Golgi lumen [78], GOLAC-1 and GOLAC-2, and may be the transporter responsible for facilitating ATP entry into the Golgi lumen [79].

ATP in the lumen also provides the ATP present in the exocytotic vesicles that mediate extracellular ATP secretion and the subsequent activation of purinergic receptors [80]. The release of ATP into extracellular fluids is well established in several physiological processes and signaling by extracellular ATP was described as early as 1929 [81]. All eukaryotic cells release ATP and most, if not all, contain plasma membrane localized nucleotide activated $\mathrm{P} 2$ receptors [82]. Since ATP is present in the cytosol of cells at concentrations up to $5 \mathrm{mM}$, any physical damage to cells will result in the release of significant amounts of ATP. Whether or not extracellular ATP could act as a co-substrate for protein kinases present in the extracellular space is still a matter of debate but will most likely occur under some physiological conditions. Several reports have described "ectokinase" activity on the surface of a number of cells including fibroblasts, neurons, myoblasts and immune cells (Reviewed in [75]). 

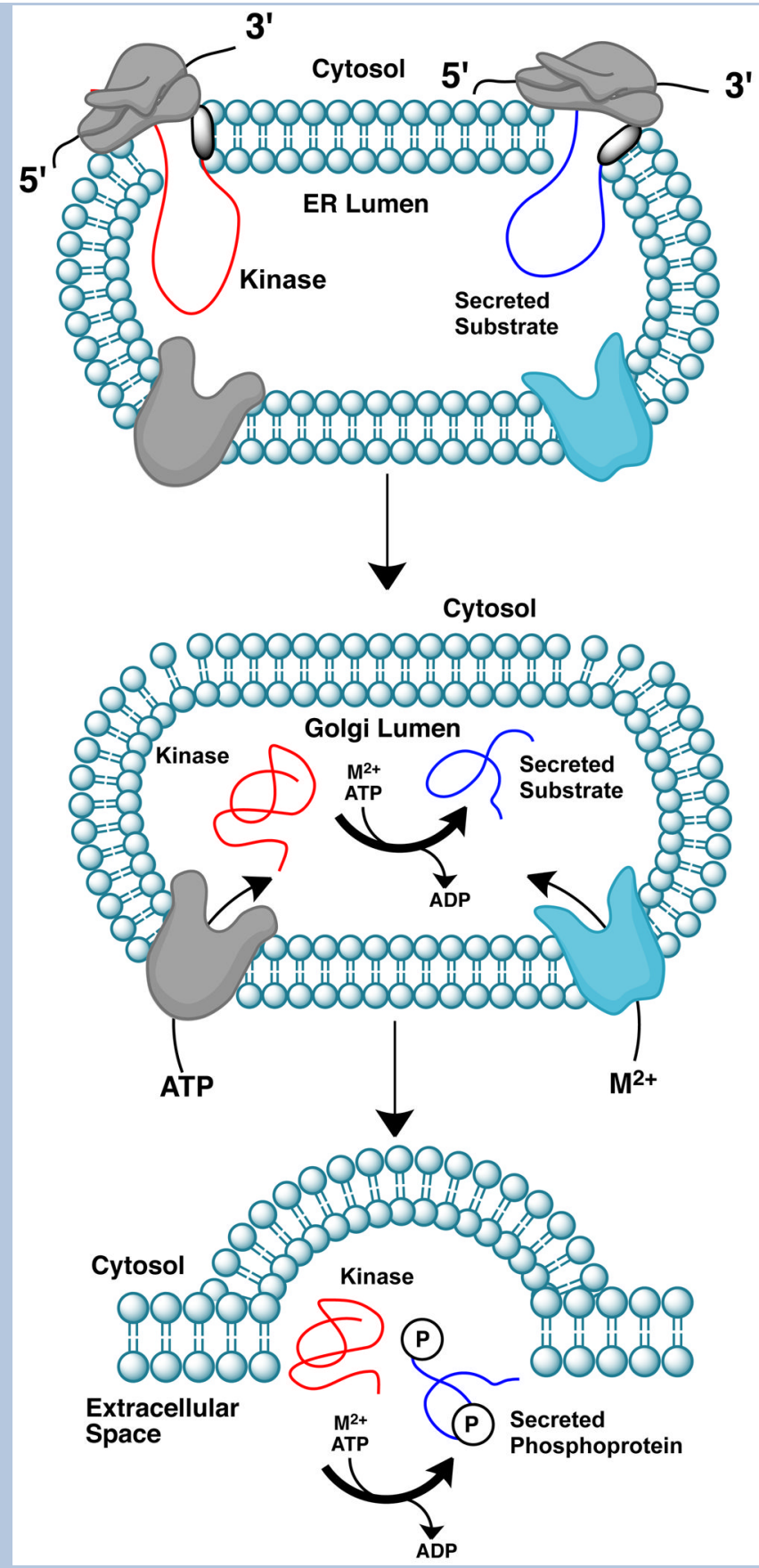

Figure I. Mechanism for the phosphorylation of extracellular proteins by secreted kinases Proteins destined for secretion have a short sequence of hydrophobic amino acids at the $\mathrm{N}$-terminus known as the signal peptide (SP, shaded black). During translation, the SPs of the kinase (red) and substrate (blue) bind the signal recognition particle embedded in the endoplasmic reticulum (ER) membrane and the nascent polypeptides enter the lumen of the ER. The signal peptidase subsequently cleaves the SP releasing the proteins from the membrane. ATP and divalent cations $\left(\mathrm{M}^{2+}\right)$ are transported into the Golgi lumen by 
specific membrane bound transporters. Catalysis may occur within the Golgi lumen or in the extracellular space.

\section{A new branch on the kinome tree}

\section{Four-jointed}

Members of the four-jointed family of proteins were shown to have weak sequence similarity to PI4KII using PI4KII as the starting point for PSI-BLAST analysis [23]. However, not one of the family members shown in Figure 1B were present in the protein kinase complement of the human genome [27]. Genetic studies in Drosophila identified four jointed (fj), as a regulator of the Hippo signaling pathway [28]. Hippo signaling controls organ size by regulating cell proliferation and apoptosis, influences planar cell polarity and is an important regulator of tumorigenesis [29]. In Drosophila, the transmembrane receptor Fat initiates the Hippo pathway in response to Dachous (Ds) binding. Both Fat and Ds are large transmembrane proteins with multiple extracellular cadherins domains. Several studies suggest that fj regulates Fat signaling control of planar cell polarity by acting non-cell autonomously and genetically upstream of Fat $[28,30]$. Therefore, Irvine and colleagues tested and found that fj could promote phosphorylation of several extracellular cadherin repeats of Fat and Ds in Drosophila S2 cells. In vitro experiments also suggested that fj was a protein kinase. Moreover, overexpression of a kinase dead version of $\mathrm{fj}$ in Drosophila abolished its biological activity [22].

$\mathrm{Fj}$ is conserved but restricted to higher vertebrates and only a limited range of invertebrates, including Drosophila and Anopheles [31]. Drosophila fj is a single pass type-II transmembrane protein whose kinase domain faces the lumen of the Golgi. Rodent and human fj (fjx1) are predicted to be secreted proteins and have been detected in the conditioned medium of several cell lines when expressed as epitope-tagged fusion proteins $[2,31,32]$. In mice, fjx 1 is expressed in the brain, the peripheral nervous system and the epithelial cells of multiple organs and its expression may be regulated by Notch signaling $[31,33]$. Fjx1 may also act as a ligand for an unknown receptor [31]. Knockout studies in mice suggest that Fjx 1 is required for the normal development of dendritic arbors in the hippocampus [34].

Fam20A

Fam20A is secreted and is differentially expressed during hematopoietic differentiation [35]. In mice, Fam 20A expression is restricted to the parathyroid gland and cells comprising the tooth such as ameloblasts and odontoblasts [36]. Mutations in Fam20A cause amelogenesis imperfecta (AI) and gingival hyperplasia syndrome in humans [37, 38]. AI is a clinically and genetically heterogeneous group of disorders of biomineralization resulting from failure of normal tooth enamel formation. AI is quite common, with incidence rates as high as 1 in 700 live births reported [39]. Enamel is the hardest and most highly mineralized tissue in the human body, consisting of $~ 95-99 \%$ mineral content, the majority of which is hydroxyapatite (HA), and $\sim 1 \%$ organic material [40]. The formation of enamel (amelogenesis) is a complex process carried out by secretory ameloblasts. These specialized cells secrete several phosphoproteins that are essential for proper enamel formation and mutations in many of these proteins causes AI [41].

Several genetic studies have implicated a role for Fam20A in AI. Using whole-exome sequencing, O'Sullivan et al. identified a homozygous nonsense mutation in exon 2 of FAM2OA in a large, consanguineous family affected by severe dental anomalies and gingival hyperplasia [37]. Resulting from this work, an additional nine AI families displaying similar phenotypes were analyzed for mutations within the FAM2OA gene. Cho 
et. al., identified three homozygous mutations in three families and a compound heterozygous mutation in one family, all of which were predicted to be loss of function mutations [38]. Fam20A knockout mice display severe AI and develop disseminated calcifications of muscular arteries and intrapulmonary calcifications without any aberrations in dentin, bone, serum calcium or serum phosphate [36]. The molecular mechanisms by which mutations in Fam20A cause AI are currently unknown but are likely due to the loss of phosphorylation of enamel-specific secreted phosphoproteins.

Fam20B

Fam20B is not a protein kinase per se; instead it phosphorylates xylose within the glycosaminoglycan-protein linkage region [42] (Figure 2). The glycosaminoglycans (GAGs) heparin sulfate (HS) and chondroitin sulfate (CS) are linear polysaccharides consisting of a repetition of [(-4GlcA $\beta 1-4 \mathrm{GlcNAca} 1-) n]$ and [(-4GlcA $\beta 1-3 \mathrm{GalNAc} \beta 1-) n]$ disaccharide units, respectively. GAGs are assembled on secreted and membrane bound core proteins through the sequential addition of monosaccharides to specific Ser residues, forming a tetrasaccharide linkage (GlcA $\beta 1-3 \mathrm{Gal} \beta 1-3 \mathrm{Gal} \beta 1-4 \mathrm{Xyl} \beta 1-O$-Ser-Core Protein) [43](Figure 2). Several modifications of the tetrasaccharide linkage have been described, including phosphorylation at the 2prime;-OH of xylose [43]. Phosphorylation appears to take place after transfer of the first galactose residue and before the addition of glucuronic acid within the lumen of the Golgi $[44,45]$. Gain and loss of function studies in HeLa cells suggest that Fam20B is a positive regulator of HS and CS formation [42]. Furthermore, a mutagenesis screen in zebrafish identified Fam20B and xylosyltransferase-1 (Xylt-1) loss-of-function mutants as having decreased cartilage matrix and increased perichondral bone formation. Genetic studies demonstrated that Fam20B and Xylt-1 operate in a linear pathway for proteoglycan production since the mutants failed to produce wild-type levels of CS [46]. In mice, deletion of the Fam2OB gene results in embryonic lethality at E13.5. Analysis of embryos revealed severe stunting with multisystem organ hypoplasia and delayed development in the skeletal system, eyes, lung, gastrointestinal tract and liver [36].

Fam20C

Fam20C (also known as dentin matrix protein-4, DMP4) is a secreted protein and appears to be the Golgi casein kinase that phosphorylates S-x-E/pS motifs within secretory pathway proteins $[2,25]$. Fam $20 \mathrm{C}$ is ubiquitously expressed with notably high levels in mineralized tissues [35, 47, 48]. Overexpression of mouse Fam20C promoted odontoblast differentiation and mineralized nodule formation [48]. Furthermore, treatment with Fam20C promoted the differentiation and mineralization of MC3T3-E1 cells [49]. Whether or not this was dependent on kinase activity was never resolved. Mutations in Fam20C in humans cause Raine Syndrome, an autosomal recessive osteosclerotic bone dysplasia first described in 1989 [50]. Raine syndrome is extremely rare with fewer than 100 cases described worldwide. Raine patients rarely survive longer than a few weeks after birth; however, a few non-lethal cases have been reported [51, 52]. Patients generally manifest with generalized osteosclerosis, ectopic calcifications, and characteristic skull and facial features including craniosynostosis, microcephaly, proptosis, exophthalmos, depressed nasal bridge, and midface hypoplasia [53]. New bone formation is also characteristic of this disorder and differentiates it from osteopetrosis (another genetic form of osteosclerosis) and other lethal and nonlethal sclerosing bone dysplasias. Loss of Fam20C in these patients' likely leads to hypophosphorylation of several secreted phosphoproteins including members of the secretory calcium binding phosphoproteins (SCPP, Figure 3) and interferes with the proper formation of HA.

In mice, global and mineralized-tissue-specific knock out of Fam20C results in notable bone lesions and hypophosphatemic rickets, but no osteosclerosis as in humans [36, 49]. The 
underlying mechanisms for the apparent discrepancies between species are unknown; however, some non-lethal cases of Raine syndrome have biochemical characteristics consistent with hypophosphatemic rickets [53]. Notably, the Fam20C null mice displayed a significant down regulation of osteoblast differentiation markers and a dramatic elevation of the phosphaturic hormone fibroblast growth factor-23 (FGF23) in serum and bone, which is likely the underlying cause of the hypophosphatemia [36, 49]. In addition to bone lesions, loss of Fam20C in mice results in several dental abnormalities and severe AI [36, 54]. Several genes involved in odontogenesis and amelogenesis were downregulated including dentin matrix protein-1 (DMP1), dentin sialophosphoprotein (DSPP), ameloblastin and amelotin [54]. Since most Raine patients do not survive long enough to develop teeth, dental abnormalities are not characteristic of the disorder. Nonetheless, dental dysplasias, including enamel defects, are present in patients with the non-lethal form of the disease [52].

\section{Fam198A and Fam198B}

Fam198A and Fam198B are found in all vertebrates but have not been functionally characterized. Mouse Fam198A localizes within the secretory pathway (ER/Golgi) and is secreted when ectopically expressed in the osteosarcoma cell line U2OS [2]. Human Fam198B (also known as Expressed in Nerve and Epithelium during Development; ENED) is a single pass Type- 2 transmembrane protein and when expressed in U2OS cells localizes within the secretory pathway but is not secreted [2]. Fam198B expression has been analyzed during development in mice and Xenopus and was also downregulated by expression of a dominant negative fibroblast growth factor [55].

\section{A laundry list of secreted phosphoproteins}

The Fam20C consensus motif, S-x-E/pS, is also phosphorylated in several proteins that are secreted by tissues other than the lactating mammary gland. Thus, Fam20C is not a mammary gland specific kinase devoted to the phosphorylation of casein, but rather a ubiquitous protein kinase dedicated to the phosphorylation of many secreted proteins within S-x-E/pS motifs. In human serum and cerebrospinal fluid, some $75 \%$ of secreted phosphoproteins contain phosphate within the Fam20C consensus motif [56-58]. Indeed, GCK activity has been detected in Golgi fractions from liver, brain, and kidney [19]. The kinase can phosphorylate secreted proteins that are not exclusively found in milk including osteopontin [59] and other members of the SCPP family. In addition, Fam20C will phosphorylate many salivary-, enamel-, dentin- and bone-specific secreted phosphoproteins (Figure 3). It appears that this family of secreted phosphoproteins descended from a common ancestor by tandem gene duplication and 23 of the 24 genes cluster on chromosome 4q13-q21 in humans (Amelogenin X is on the $X$ chromosome)[60] As the name suggests, members of the SCPP family are secreted phosphoproteins (phosphorylated on multiple $\mathrm{S}-\mathrm{x}-\mathrm{E} / \mathrm{S}$ motifs), have a high affinity for calcium ions and regulate biomineralization. The small integrin-binding ligand, N-linked glycoproteins (SIBLINGs) comprise a subfamily of the SCPPs consisting of five identically oriented tandem genes clustered within an $\sim 375 \mathrm{~kb}$ span of nucleotides on human chromosome 4 . The genes encode DMP1, DSPP, osteopontin (OPN), matrix extracellular phosphoglycoprotein (MEPE) and bone sialoprotein (BSP), all of which contain multiple phosphorylated S-x-E/S motifs and are phosphorylated by Fam20C [2, 25]. The SIBLINGs have well-documented roles in regulating HA formation in bone and dentin and in many instances, regulation is known to be dependent upon phosphorylation [61]. These results suggest an important role for Fam20C in biomineralization.

Many secreted proteins without any apparent link to biomineralization are also phosphorylated within S-x-E/S motifs (Table 2). The stoichiometry of phosphorylation and the physiological functions of these phosphorylation events are - in most cases - 
unknown. The oocyte secreted factor bone morphogenetic protein-15 (BMP-15) is a substrate for G-CK and the phosphorylation of BMP-15 is essential for its bioactivity [62, 63]. Insulin-like growth factor binding protein-1 (IGFBP-1) in human amniotic fluid is phosphorylated on five serines, all of which conform to the Fam20C consensus site [64]. Phosphorylation of IGFBP-1 enhances its binding to insulin-like growth factor-1 (IGF-1), which reduces the availability of free IGF-1 to interact with its receptor [65]. Coagulation factor IX is phosphorylated in human plasma within an S-x-E motif, which is likely important for its biological activity as the nonphosphorylated form rapidly disappears from plasma [66]. Several neuropeptide hormones, cytokines, proteases and extracellular matrix components are also putative Fam20C substrates (Table 2). Furthermore, proteins in the secretory pathway that are not secreted may also serve as substrates for Fam20C, including ER and Golgi resident proteins, and extracellular domains of cell surface receptors. Indeed, some $10 \%$ of the human phosphoproteome contains phosphate within the Fam20C consensus motif (Figure 4). This percentage may over-represent the number of substrates for Fam20C because not all proteins containing phosphate on S-X-E motifs will localize within the secretory pathway. In any event, these observations suggest that Fam20C will likely have many substrates in addition to proteins involved in biomineralization.

\section{Secreted kinases and human disease}

To date, the only secreted kinases that have been associated with human disease are Fam20A ad Fam20C. Mutations in Fam20C cause Raine Syndrome in humans and hypophosphatemic rickets in mice. We propose that loss of Fam20C leads to these disorders of biomineralization by interfering with proper HA formation as a result of the loss of phosphorylation of SCPPs and possibly other secreted proteins. One mechanism may be that loss of phosphorylation of the SCPPs prevents the sequestration of calcium, thereby allowing free calcium to bind inorganic phosphate, reach supersaturation and precipitate as HA. A somewhat similar situation exists in milk where the casein micelle acts to fluidize the casein molecules and solubilize the calcium and inorganic phosphate. When the concentration of these two ions are quantified in milk containing $>2 \%$ protein, the levels are supersaturated and would, by themselves, yield insoluble precipitates [67]. However, not all SCPPs inhibit the calcification process since many members play important roles in promoting tissue mineralization. Thus, the SCPPs appear to differentially regulate growth kinetics and crystal size in a manner that is highly dependent on phosphorylation [61]. Furthermore, the SCPPs are proteolytically processed and phosphorylation may regulate this process. Additionally, phosphorylation of the SIBLINGs may influence binding to cell surface integrin receptors. The SIBLINGs have conserved Arg-Gly-Asp (RGD) integrin binding motifs and function as signal transducers to promote cell adhesion, motility and survival.

As for the detailed molecular events that contribute to the pathogenesis of Raine Syndrome and AI, further work is needed to establish which SCPP members are most sensitive to the loss of phosphorylation by Fam20C and which sites of phosphorylation are most important. Unquestionably, the most informative example of SCPP phosphorylation-dependent regulation of biomineralization comes from genetic studies of a family with AI. Several family members had a novel missense mutation (g.12573C $>$ T) that substituted Ser for Leu (p.S216L) in the 32-kDa enamelin cleavage product. Curiously, this Ser lies within the Fam20C consensus, $\mathrm{S}-\mathrm{x}-\mathrm{E}$, and is phosphorylated in native enamelin. Thus, loss of phosphorylation of enamelin by Fam20C is sufficient to cause abnormal enamel mineralization and the human disease AI [68]. Furthermore, mutations in the SCPP amelogenin-X (AMELX) also cause AI [41]. AMELX has one phospho-Ser within the Fam20C consensus motif that plays an important role in regulating mineralization by inhibiting calcium phosphate precipitation [69]. As discussed above, loss of Fam20C or 
Fam20A causes AI in mice and humans [36-38]. How loss of Fam20A results in AI is not known but may also be due to the lack of phosphorylation of secreted substrates important for proper enamel formation. However, the substrates for Fam20A are unknown and may be distinct from Fam20C substrates. In any event, both kinases appear to be essential for proper enamel formation and may have non-redundant roles and/or operate in a linear pathway crucial for proper enamel formation. Clearly, Fam20A and Fam20C are involved in a previously unrecognized aspect of enamel formation. Future work in this area may lead to novel therapeutics for patients with AI and will certainly advance our understanding of the molecular events underlying enamel formation.

\section{Concluding remarks}

The four-jointed family of secreted protein kinases has expanded the repertoire of the human kinome and solved a perplexing mystery that dates back to the nineteeth century when Hammarsten detected phosphorous in casein. Importantly, this discovery has established functions for two genes, Fam20A and Fam20C, which are implicated in the human diseases Amelogenesis Imperfecta and Raine Syndrome, respectively. Much remains to be learned, including how phosphorylation affects the function of secreted proteins and how these phosphorylation events contribute to human physiology and/or pathophysiology. Moreover, what are the substrates for Fam20A, Fam198A, and Fam198B (Box 3)? Does human fjx1 phosphorylate the extracellular domains of cadherins in mammals? The physiological significance of secreted protein phosphorylation of substrates that are not involved in biomineralization also warrants further investigation. It will be imperative to determine the stoichiometry and ultimately the physiological consequence of phosphorylation within these secreted phosphoproteins. As mentioned above, a small number of secreted phosphoproteins have defined functions that are dependent on phosphorylation. However, for the majority of secreted phosphoproteins, established functions for phosphorylation have not been elucidated.

\section{Box 3}

\section{What about the other secreted kinases?}

An important question moving forward is: What are the substrates for the other secreted protein kinases? As a first step it will be important to determine if the kinases are catalytically active. If so, determining a consensus motif that they phosphorylate will facilitate the search for physiological substrates. Since the generation of $\sim 75 \%$ of the CSF and serum phosphoproteome may be attributed to Fam20C, the obvious next step will be to test if the remaining $25 \%$ of secreted phosphoproteins are substrates for other secreted protein kinases. DSPP, for example, contains $\sim 200$ phosphoserines and is quite possibly the most highly phosphorylated protein in humans. The motif surrounding these phosphoserines rarely conforms to the Fam20C consensus motif (196 are S-D-S motifs in human DSPP). Unless however phosphorylation by an additional protein kinase "primes" DSPP by creating a S-x-pS Fam20C recognition motif. Phosphorylation of DSPP would therefore occur in a hierarchal manner [83]. Furthermore, there may be other specificity determinants for Fam20C in addition to the S-x-E/pS motif. Indeed, the GEF-CK can phosphorylate proline-rich phosphoprotein (PRP-1) on a Ser residue within the S-E-Q-FI-E-D-D sequence. Using a series of peptides, it was shown that its kinase activity was dependent on the Gln at the $\mathrm{N}+2$ position and on three acidic residues at the $\mathrm{N}+5, \mathrm{~N}+6$ and $\mathrm{N}+7$ positions, thus defining a novel consensus sequence for Fam20C: S-x-Q-x-x-E/ D-E/D-E/D [84]. 
The phosphorylation of secreted S-x-E/pS containing proteins, including the SCPPs, by Fam20C; phosphorylation of xylose within the glycosaminoglycan-protein linkage region by Fam20B; and phosphorylation of atypical cadherins by four-jointed has set the stage for the emerging new field of extracellular protein phosphorylation by secreted protein kinases. Nearly 130 years of protein phosphorylation research have uncovered many functions for intracellular phosphorylation, such as altering enzyme activity, modifying binding affinity, and altering stability. There is no reason to believe that the fundamental mechanisms governing intracellular protein phosphorylation will be any different for extracellular protein phosphorylation. Future work will undoubtedly uncover unappreciated aspects of extracellular protein phosphorylation.

\section{Acknowledgments}

Supported in part by National Institutes of Health (NIH) Grants DK018849-36 and DK018024-37 (to J.E.D.), NIH/ National Cancer Institute Training Grant T32 CA009523 (to V.S.T.) and AIRC (Associazione Italiana per la Ricerca sul Cancro) Grant IG-10312 (to L.A.P.). We thank Claudia Dixon and Carolyn Worby for insightful discussions regarding the manuscript. We would also like to apologize to colleagues whose work we were not able to cite due to space constraints.

\section{Glossary}

Protein kinase

Casein

Signal peptide (SP)

Golgi Casein Kinase

(G-CK, recently

identified as

Fam20C)

$\beta(28-40)$

Hydroxyapatite (HA)

Osteosclerosis

Odontoblasts

Ameloblasts

Raine Syndrome

Amelogenesis imperfecta (AI) an enzyme that transfers phosphate (usually from ATP) to protein substrates

An abundant phosphoprotein found in milk that, in 1883, was the first protein shown to contain phosphate

a short sequence of hydrophobic amino acids that directs newly synthesized proteins to the secretory pathway

A protein kinase detected in highly enriched Golgi fractions from lactating mammary glands and other tissues that phosphorylates casein and many secreted proteins within S-x-E/pS motifs. The kinase activity was detected in 1972; however the discovery that the G-CK was Fam20C would take nearly 40 years

a synthetic peptide corresponding to a sequence in bovine $\beta$ casein that is used to assay Fam20C/G-CK activity with absolute specificity

a naturally occurring calcium-phosphate complex with the formula $\mathrm{Ca}_{5}\left(\mathrm{PO}_{4}\right)_{3}(\mathrm{OH})$. HA is the main structural component of vertebrate bone

An increase in the density of bone

a specialized cell found on the outer surface of the dental pulp that functions to produce dentin, a major component of teeth

specialized cells that are present during tooth development and deposit enamel

A rare, usually fatal, autosomal recessive osteosclerotic bone dysplasia. Raine Syndrome is caused by mutations in Fam20C

a heterogeneous group of tooth development disorders in which the teeth are covered with thin, abnormally formed enamel. Mutations in several genes, including Fam20A, cause AI 


\section{References}

1. Hammarsten O. Zur Frage ob Caseín ein einheitlicher Stoff sei. Hoppe-Seyler's Zeitschrift für Physiologische Chemie. 1883:227-273.

2. Tagliabracci VS, et al. Secreted kinase phosphorylates extracellular proteins that regulate biomineralization. Science. 2012; 336:1150-1153. [PubMed: 22582013]

3. Lipmann F. Über die Bindung der Phosphorsäre in Phosphorproteinen. I. Biochem Z. 1933; 262:3.

4. Lipmann FA, Levene PA. Serinephosphoric acid obtained on hydrolysis of vitellinic acid. J Biol Chem. 1932; 98:109-114.

5. Burnett G, Kennedy EP. The enzymatic phosphorylation of proteins. J Biol Chem. 1954; 211:969980. [PubMed: 13221602]

6. Krebs EG, Fischer EH. The phosphorylase b to a converting enzyme of rabbit skeletal muscle. Biochim Biophys Acta. 1956; 20:150-157. [PubMed: 13315361]

7. Rabinowitz M, Lipmann F. Reversible phosphate transfer between yolk phosphoprotein and adenosine triphosphate. J Biol Chem. 1960; 235:1043-1050. [PubMed: 14435647]

8. Walsh DA, et al. An adenosine $3^{\prime}, 5^{\prime}$-monophosphate-dependant protein kinase from rabbit skeletal muscle. J Biol Chem. 1968; 243:3763-3765. [PubMed: 4298072]

9. Pinna LA, et al. Isolation and properties of a protein kinase from rat liver microsomes. Biochim Biophys Acta. 1969; 178:199-201. [PubMed: 5773455]

10. Rodnight R, Lavin BE. Phosvitin kinase from brain: activation by ions and subcellular distribution. Biochem J. 1964; 93:84-91. [PubMed: 4284465]

11. Allende JE, Allende CC. Protein kinases. 4. Protein kinase CK2: an enzyme with multiple substrates and a puzzling regulation. FASEB J. 1995; 9:313-323. [PubMed: 7896000]

12. Bingham EW, et al. Phosphorylation of casein. Role of the golgi apparatus. J Biol Chem. 1972; 247:8193-8194. [PubMed: 4629742]

13. Moore A, et al. Purification and tissue-specific expression of casein kinase from the lactating guinea-pig mammary gland. Eur J Biochem. 1985; 152:729-737. [PubMed: 3863754]

14. Mercier JC. Phosphorylation of caseins, present evidence for an amino acid triplet code posttranslationally recognized by specific kinases. Biochimie. 1981; 63:1-17. [PubMed: 7011421]

15. Mercier JC, et al. Primary structure of bovine s1 casein. Complete sequence. Eur J Biochem. 1971; 23:41-51. [PubMed: 4331376]

16. Lasa-Benito M, et al. Golgi apparatus mammary gland casein kinase: monitoring by a specific peptide substrate and definition of specificity determinants. FEBS Lett. 1996; 382:149-152. [PubMed: 8612738]

17. Meggio F, et al. Synthetic fragments of beta-casein as model substrates for liver and mammary gland casein kinases. Eur J Biochem. 1989; 186:459-464. [PubMed: 2606100]

18. Meggio F, et al. Substrate-specificity determinants for a membrane-bound casein kinase of lactating mammary gland. A study with synthetic peptides. Eur J Biochem. 1988; 177:281-284. [PubMed: 3191919]

19. Lasa M, et al. Rat liver Golgi apparatus contains a protein kinase similar to the casein kinase of lactating mammary gland. Eur J Biochem. 1997; 243:719-725. [PubMed: 9057837]

20. Duncan JS, et al. Purification of Golgi casein kinase from bovine milk. Biochem J. 2000; 350(Pt 2):463-468. [PubMed: 10947960]

21. Pouliot Y, Karp PD. A survey of orphan enzyme activities. BMC Bioinformatics. 2007; 8:244. [PubMed: 17623104]

22. Ishikawa HO, et al. Four-jointed is a Golgi kinase that phosphorylates a subset of cadherin domains. Science. 2008; 321:401-404. [PubMed: 18635802]

23. Barylko B, et al. A novel family of phosphatidylinositol 4-kinases conserved from yeast to humans. J Biol Chem. 2001; 276:7705-7708. [PubMed: 11244087]

24. Correia FF, et al. Kinase activity of overexpressed HipA is required for growth arrest and multidrug tolerance in Escherichia coli. J Bacteriol. 2006; 188:8360-8367. [PubMed: 17041039]

25. Ishikawa HO, et al. The Raine Syndrome Protein FAM20C Is a Golgi Kinase That Phosphorylates Bio-Mineralization Proteins. PLoS One. 2012; 7:e42988. [PubMed: 22900076] 
26. Lolli G, et al. Inhibition of Protein Kinase CK2 by Flavonoids and Tyrphostins. A Structural Insight. Biochemistry. 2012; 51:6097-6107. [PubMed: 22794353]

27. Manning G, et al. The protein kinase complement of the human genome. Science. 2002; 298:19121934. [PubMed: 12471243]

28. Yang $\mathrm{CH}$, et al. Regulation of Frizzled by fat-like cadherins during planar polarity signaling in the Drosophila compound eye. Cell. 2002; 108:675-688. [PubMed: 11893338]

29. Zhao B, et al. The Hippo-YAP pathway in organ size control and tumorigenesis: an updated version. Genes Dev. 2010; 24:862-874. [PubMed: 20439427]

30. Cho E, et al. Delineation of a Fat tumor suppressor pathway. Nat Genet. 2006; 38:1142-1150. [PubMed: 16980976]

31. Rock R, et al. Fjx1: a notch-inducible secreted ligand with specific binding sites in developing mouse embryos and adult brain. Dev Dyn. 2005; 234:602-612. [PubMed: 16145673]

32. Bendtsen JD, et al. Improved prediction of signal peptides: SignalP 3.0. J Mol Biol. 2004; 340:783-795. [PubMed: 15223320]

33. Ashery-Padan R, et al. Fjx1, the murine homologue of the Drosophila four-jointed gene, codes for a putative secreted protein expressed in restricted domains of the developing and adult brain. Mech Dev. 1999; 80:213-217. [PubMed: 10072791]

34. Probst B, et al. The rodent Four-jointed ortholog Fjx1 regulates dendrite extension. Dev Biol. 2007; 312:461-470. [PubMed: 18028897]

35. Nalbant D, et al. FAM20: an evolutionarily conserved family of secreted proteins expressed in hematopoietic cells. BMC Genomics. 2005; 6:11. [PubMed: 15676076]

36. Vogel P, et al. Amelogenesis Imperfecta and Other Biomineralization Defects in Fam20a and Fam20c Null Mice. Vet Pathol. 2012; 49:998-1017. [PubMed: 22732358]

37. O'Sullivan J, et al. Whole-Exome sequencing identifies FAM20A mutations as a cause of amelogenesis imperfecta and gingival hyperplasia syndrome. Am J Hum Genet. 2011; 88:616620. [PubMed: 21549343]

38. Cho SH, et al. Novel FAM20A mutations in hypoplastic amelogenesis imperfecta. Hum Mutat. 2012; 33:91-94. [PubMed: 21990045]

39. Backman B, Holm AK. Amelogenesis imperfecta: prevalence and incidence in a northern Swedish county. Community Dent Oral Epidemiol. 1986; 14:43-47. [PubMed: 3456873]

40. Eastoe JE. Organic matrix of tooth enamel. Nature. 1960; 187:411-412. [PubMed: 13819116]

41. Stephanopoulos G, et al. Genes and related proteins involved in amelogenesis imperfecta. J Dent Res. 2005; 84:1117-1126. [PubMed: 16304440]

42. Koike T, et al. FAM20B is a kinase that phosphorylates xylose in the glycosaminoglycan-protein linkage region. Biochem J. 2009; 421:157-162. [PubMed: 19473117]

43. Bishop JR, et al. Heparan sulphate proteoglycans fine-tune mammalian physiology. Nature. 2007; 446:1030-1037. [PubMed: 17460664]

44. Gulberti S, et al. Phosphorylation and sulfation of oligosaccharide substrates critically influence the activity of human beta1,4-galactosyltransferase 7 (GalT-I) and beta1,3-glucuronosyltransferase I (GlcAT-I) involved in the biosynthesis of the glycosaminoglycan-protein linkage region of proteoglycans. J Biol Chem. 2005; 280:1417-1425. [PubMed: 15522873]

45. Tone Y, et al. 2-o-phosphorylation of xylose and 6-o-sulfation of galactose in the protein linkage region of glycosaminoglycans influence the glucuronyltransferase-I activity involved in the linkage region synthesis. J Biol Chem. 2008; 283:16801-16807. [PubMed: 18400750]

46. Eames BF, et al. Mutations in fam $20 \mathrm{~b}$ and xylt1 reveal that cartilage matrix controls timing of endochondral ossification by inhibiting chondrocyte maturation. PLoS Genet. 2011; 7:e1002246. [PubMed: 21901110]

47. Wang X, et al. Expression of FAM20C in the osteogenesis and odontogenesis of mouse. J Histochem Cytochem. 2010; 58:957-967. [PubMed: 20644212]

48. Hao J, et al. Dentin matrix protein 4, a novel secretory calcium-binding protein that modulates odontoblast differentiation. J Biol Chem. 2007; 282:15357-15365. [PubMed: 17369251]

49. Wang X, et al. Inactivation of a novel FGF23 regulator, FAM20C, leads to hypophosphatemic rickets in mice. PLoS Genet. 2012; 8:e1002708. [PubMed: 22615579] 
50. Raine J, et al. Unknown syndrome: microcephaly, hypoplastic nose, exophthalmos, gum hyperplasia, cleft palate, low set ears, and osteosclerosis. J Med Genet. 1989; 26:786-788. [PubMed: 2614802]

51. Simpson MA, et al. Mutations in FAM20C also identified in non-lethal osteosclerotic bone dysplasia. Clin Genet. 2009; 75:271-276. [PubMed: 19250384]

52. Fradin M, et al. Osteosclerotic bone dysplasia in siblings with a Fam20C mutation. Clin Genet. 2011; 80:177-183. [PubMed: 20825432]

53. Simpson MA, et al. Mutations in FAM20C are associated with lethal osteosclerotic bone dysplasia (Raine syndrome), highlighting a crucial molecule in bone development. Am J Hum Genet. 2007; 81:906-912. [PubMed: 17924334]

54. Wang X, et al. FAM20C plays an essential role in the formation of murine teeth. J Biol Chem. 2012; 287:35934-35942. [PubMed: 22936805]

55. Meszaros R, et al. Expression of the novel gene Ened during mouse and Xenopus embryonic development. Int J Dev Biol. 2008; 52:1119-1122. [PubMed: 18956345]

56. Salvi M, et al. Motif analysis of phosphosites discloses a potential prominent role of the Golgi casein kinase (GCK) in the generation of human plasma phospho-proteome. J Proteome Res. 2010; 9:3335-3338. [PubMed: 20450225]

57. Bahl JM, et al. Characterization of the human cerebrospinal fluid phosphoproteome by titanium dioxide affinity chromatography and mass spectrometry. Anal Chem. 2008; 80:6308-6316. [PubMed: 18702456]

58. Zhou W, et al. An initial characterization of the serum phosphoproteome. J Proteome Res. 2009; 8:5523-5531. [PubMed: 19824718]

59. Lasa M, et al. Phosphorylation of osteopontin by Golgi apparatus casein kinase. Biochem Biophys Res Commun. 1997; 240:602-605. [PubMed: 9398611]

60. Kawasaki K. The SCPP gene family and the complexity of hard tissues in vertebrates. Cells Tissues Organs. 2011; 194:108-112. [PubMed: 21576905]

61. George A, Veis A. Phosphorylated proteins and control over apatite nucleation, crystal growth, and inhibition. Chem Rev. 2008; 108:4670-4693. [PubMed: 18831570]

62. McMahon HE, et al. Phosphorylation of bone morphogenetic protein-15 and growth and differentiation factor-9 plays a critical role in determining agonistic or antagonistic functions. Endocrinology. 2008; 149:812-817. [PubMed: 18006624]

63. Tibaldi E, et al. Golgi apparatus casein kinase phosphorylates bioactive Ser-6 of bone morphogenetic protein 15 and growth and differentiation factor 9. FEBS Lett. 2010; 584:801-805. [PubMed: 20067794]

64. Dolcini L, et al. Identification of the amniotic fluid insulin-like growth factor binding protein-1 phosphorylation sites and propensity to proteolysis of the isoforms. FEBS J. 2009; 276:60336046. [PubMed: 19765076]

65. Jones JI, Clemmons DR. Insulin-like growth factors and their binding proteins: biological actions. Endocr Rev. 1995; 16:3-34. [PubMed: 7758431]

66. Atoda $\mathrm{H}$, et al. Characterization of a monoclonal antibody B1 that recognizes phosphorylated Ser-158 in the activation peptide region of human coagulation factor IX. J Biol Chem. 2006; 281:9314-9320. [PubMed: 16467297]

67. Farrell HM Jr, et al. The caseins of milk as calcium-binding proteins. Methods Mol Biol. 2002; 172:97-140. [PubMed: 11833362]

68. Chan HC, et al. Altered enamelin phosphorylation site causes amelogenesis imperfecta. J Dent Res. 2010; 89:695-699. [PubMed: 20439930]

69. Kwak SY, et al. Role of 20-kDa amelogenin (P148) phosphorylation in calcium phosphate formation in vitro. J Biol Chem. 2009; 284:18972-18979. [PubMed: 19443653]

70. Colaert N, et al. Improved visualization of protein consensus sequences by iceLogo. Nat Methods. 2009; 6:786-787. [PubMed: 19876014]

71. Mauro Salvi LC, Pinna Lorenzo A. Variable contribution of protein kinases to the generation of the human phosphoproteome: a global weblogo analysis. Biomolecular Concepts. 2010; 1:185-195. 
72. Meggio F, Pinna LA. Phosphorylation of phosvitin by casein kinase-2 provides the evidence that phosphoserines can replace carboxylic amino acids as specificity determinants. Biochim Biophys Acta. 1988; 971:227-231. [PubMed: 2901861]

73. Levene PA, Alsberg C. Zur chemie der paranucleinsaure. Hoppe-Seyler's Z Physiol Chemie. 1900; 31:543-555.

74. Capasso JM, et al. Mechanism of phosphorylation in the lumen of the Golgi apparatus. Translocation of adenosine $5^{\prime}$-triphosphate into Golgi vesicles from rat liver and mammary gland. J Biol Chem. 1989; 264:5233-5240. [PubMed: 2925690]

75. Redegeld FA, et al. Ecto-protein kinases: ecto-domain phosphorylation as a novel target for pharmacological manipulation? Trends Pharmacol Sci. 1999; 20:453-459. [PubMed: 10542445]

76. Puglielli L, et al. Identification, purification, and characterization of the rat liver golgi membrane ATP transporter. J Biol Chem. 1999; 274:12665-12669. [PubMed: 10212247]

77. Maeda Y, et al. GPHR is a novel anion channel critical for acidification and functions of the Golgi apparatus. Nat Cell Biol. 2008; 10:1135-1145. [PubMed: 18794847]

78. Thompson RJ, et al. Anion channels transport ATP into the Golgi lumen. Am J Physiol Cell Physiol. 2006; 290:C499-514. [PubMed: 16403948]

79. Caldwell JH, Howell KE. Pores galore for the Golgi. Nat Cell Biol. 2008; 10:1125-1126. [PubMed: 18830217]

80. Zhong X, et al. ATP uptake in the Golgi and extracellular release require Mcd4 protein and the vacuolar H+-ATPase. J Biol Chem. 2003; 278:33436-33444. [PubMed: 12807869]

81. Drury AN, Szent-Gyorgyi A. The physiological activity of adenine compounds with especial reference to their action upon the mammalian heart. J Physiol. 1929; 68:213-237. [PubMed: 16994064]

82. Corriden R, Insel PA. Basal release of ATP: an autocrine-paracrine mechanism for cell regulation. Sci Signal. 2010; 3:re1. [PubMed: 20068232]

83. Roach PJ. Multisite and hierarchal protein phosphorylation. J Biol Chem. 1991; 266:14139-14142. [PubMed: 1650349]

84. Brunati AM, et al. Novel consensus sequence for the Golgi apparatus casein kinase, revealed using proline-rich protein-1 (PRP1)-derived peptide substrates. Biochem J. 2000; 351(Pt 3):765-768. [PubMed: 11042132]

85. May LT, Sehgal PB. Phosphorylation of interleukin-6 at serine54: an early event in the secretory pathway in human fibroblasts. Biochem Biophys Res Commun. 1992; 185:524-530. [PubMed: 1610348]

86. Dewpura T, et al. PCSK9 is phosphorylated by a Golgi casein kinase-like kinase ex vivo and circulates as a phosphoprotein in humans. FEBS J. 2008; 275:3480-3493. [PubMed: 18498363]

87. Tang J, et al. Amino-acid sequence of porcine pepsin. Proc Natl Acad Sci U S A. 1973; 70:34373439. [PubMed: 4587252]

88. Blombaeck B, et al. Amino-acid sequence and the occurrence of phosphorus in human fibrinopeptides. Nature. 1962; 193:833-834. [PubMed: 13870090]

89. Dockray GJ, et al. Post-translational processing of the porcine gastrin precursor by phosphorylation of the COOH-terminal fragment. J Biol Chem. 1987; 262:8643-8647. [PubMed: 3597391]

90. Lee $\mathrm{SN}$, et al. Neuroendocrine protein 7B2 can be inactivated by phosphorylation within the secretory pathway. J Biol Chem. 2006; 281:3312-3320. [PubMed: 16286464]

91. Bennett HP, et al. Biosynthesis of phosphorylated forms of corticotropin-related peptides. Proc Natl Acad Sci U S A. 1981; 78:4713-4717. [PubMed: 6272271]

92. Giorgianni F, et al. Identification and characterization of phosphorylated proteins in the human pituitary. Proteomics. 2004; 4:587-598. [PubMed: 14997482]

93. Gadroy P, et al. Phosphorylation and O-glycosylation sites of human chromogranin A (CGA79439) from urine of patients with carcinoid tumors. J Biol Chem. 1998; 273:34087-34097. [PubMed: 9852066]

94. Beranova-Giorgianni S, et al. Phosphoproteomic analysis of the human pituitary. Pituitary. 2006; 9:109-120. [PubMed: 16807684] 
95. Strub JM, et al. Phosphorylation and O-glycosylation sites of bovine chromogranin A from adrenal medullary chromaffin granules and their relationship with biological activities. J Biol Chem. 1997; 272:11928-11936. [PubMed: 9115255]

96. Watkinson A, et al. The isolation and chemical characterization of phosphorylated enkephalincontaining peptides from bovine adrenal medulla. J Biol Chem. 1989; 264:3061-3065. [PubMed: 2914943] 
A

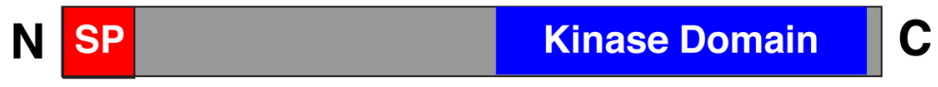

B

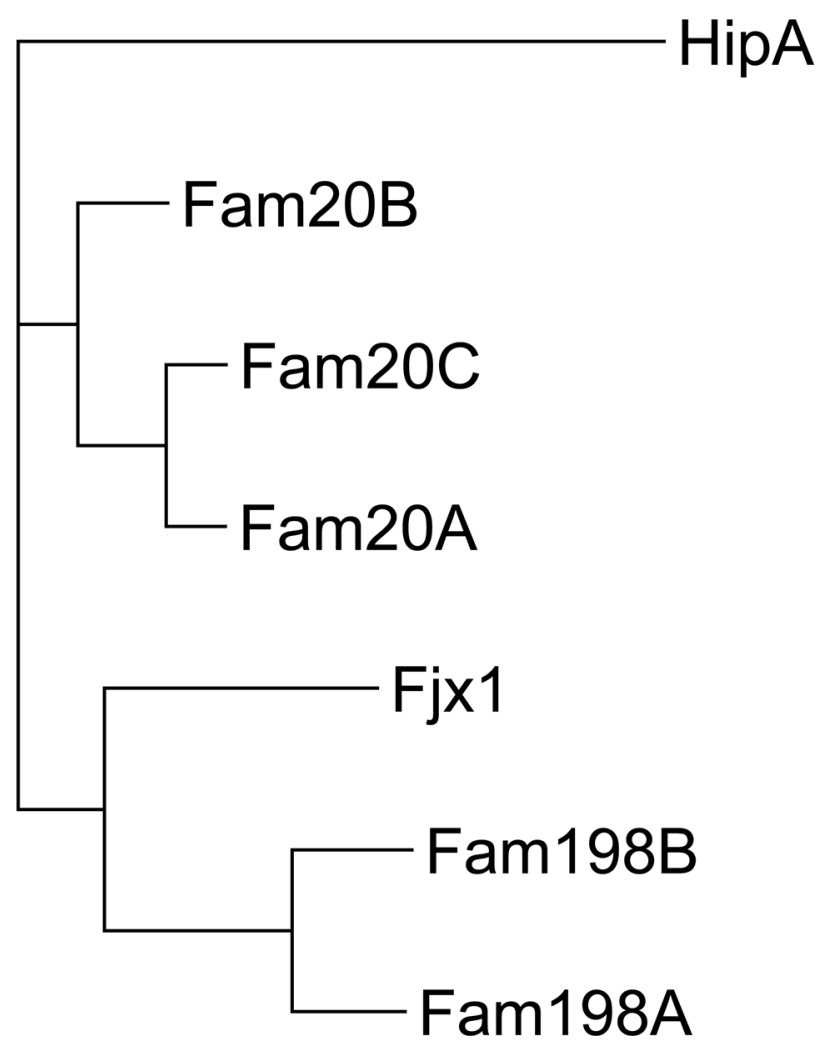

Figure 1. Four-jointed family of protein kinases

(A) The architecture of a secreted protein kinase consists of an N-terminal SP, a C-terminal kinase domain and no transmembrane domain. (B) A phylogenetic tree depicting the human four-jointed family of protein kinases (Fjx1, Fam20A, Fam20B, Fam20C, Fam198A and Fam198B). The bacterial kinase, HipA shows weak sequence similarity to the four-jointed family of protein kinases. 


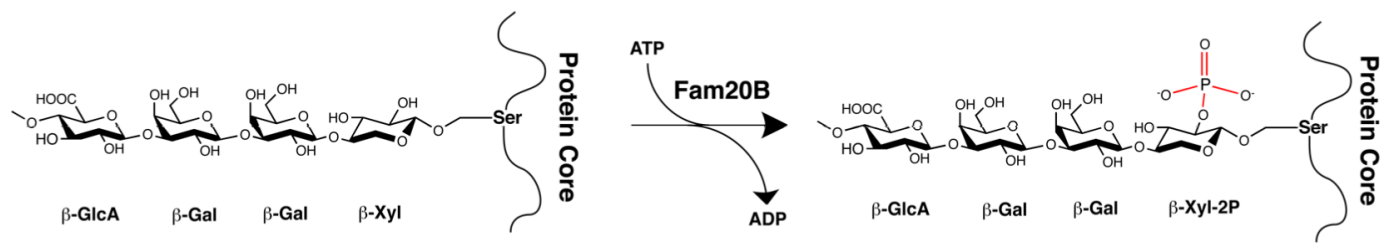

Figure 2. Fam20B phosphorylates xylose within the glycosaminoglycan-protein linkage region Glycosaminoglycans are synthesized by the attachment of a tetrasaccharide linkage to specific Ser residues within core proteins during transit through the secretory pathway. The tetrasaccharide consists of the monosaccharides xylose (Xyl), galactose $(\mathrm{Gal})$, and glucuronic acid (GlcA) linked by glucosidic bonds in a GlcA $\beta 1-3 \mathrm{Gal} \beta 1-3 \mathrm{Gal} \beta 1-4 \mathrm{Xyl} \beta 1$ orientation. Fam20B transfers a phosphate from ATP to the $2^{\prime}-\mathrm{OH}$ of xylose $(\beta-\mathrm{Xyl})$, which may regulate heparin and chondroitin sulfate formation. 


\section{Pro/GIn-rich SCPPs}

\section{Acidic SCPPs}

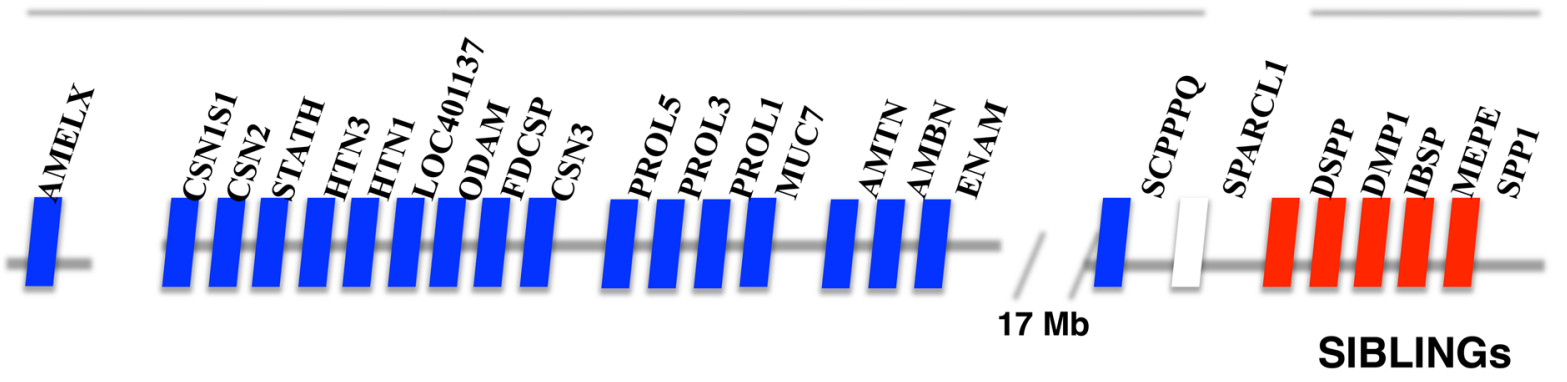

Figure 3. The secretory calcium binding phosphoprotein (SCPP) family

Shown is the small region on human chromosome 4 in which 23 of the 24 SCPP family members are located. Amelogenin X is on the X chromosome. The SCPPs are secreted phosphoproteins (phosphorylated on multiple $\mathrm{S}-\mathrm{X}-\mathrm{E} / \mathrm{S}$ motifs), have a high affinity for calcium ions and regulate biomineralization. The Pro/GIn rich SCPPs and the acidic SIBLING SCPPs are shown in blue and red, respectively Adapted from [60]. 


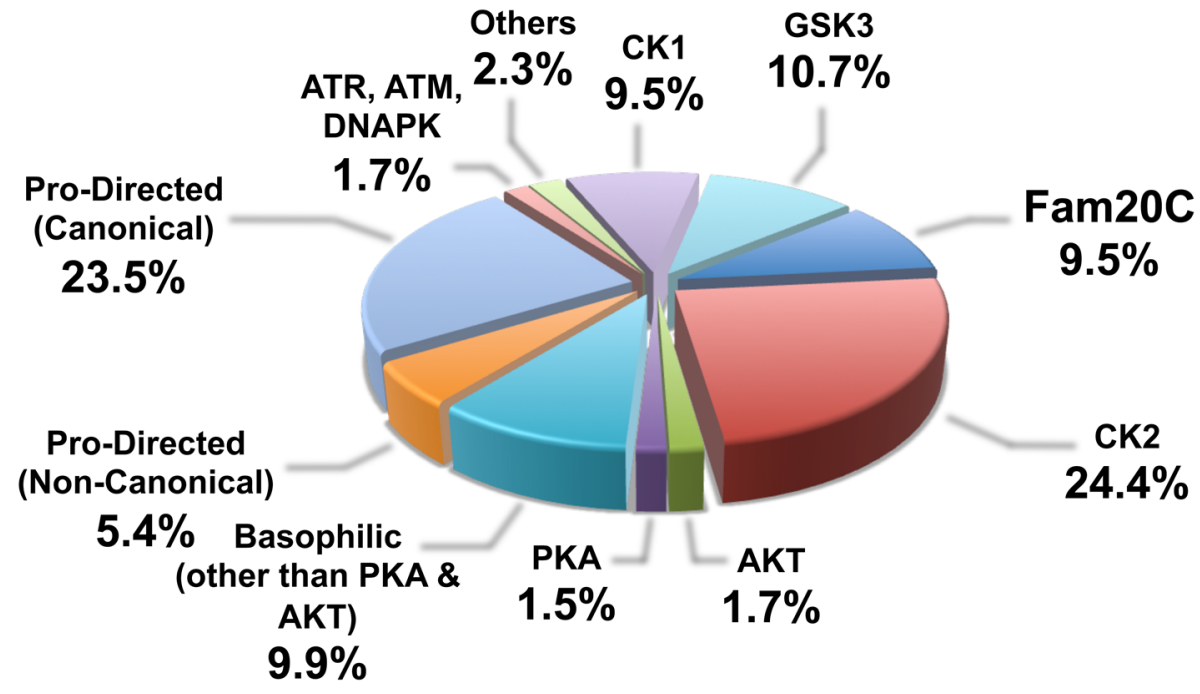

Figure 4. Relevant contribution of Fam20C to the generation of the human phosphoproteome Data have been extrapolated from $35679 \mathrm{Ser} / \mathrm{Thr}$ phosphosites available in the PhosphoSitePlus by performing a two sample logo ("ice-logo") analysis [70] for each kinase as described by Salvi et al. [71]. In the case of Fam20C, only phosphosites conforming to the consensus $\mathrm{S}-\mathrm{x}-\mathrm{E} / \mathrm{pS}$ but not to that of CK2 (S/T-X-X-E/D/pS) have been considered. 
Table 1

Casein phosphorylation through the years.

\begin{tabular}{|c|l|c|}
\hline Year & Event & Ref \\
\hline 1883 & Casein is a phosphoprotein & {$[1]$} \\
\hline 1900 & Vitellinic acid/phosvitin is a phosphoprotein & {$[73]$} \\
\hline 1932 & Phosphoserine in vitellinic acid phosvitin & {$[4]$} \\
\hline 1933 & Phosphoserine in casein & {$[3]$} \\
\hline 1954 & Casein is used as a model substrate for the detection of the first protein kinase activity & {$[5]$} \\
\hline 1960 & Casein/phosvitin kinases are distinct from phosphorylase kinase & {$[7]$} \\
\hline 1969 & Two distinct casein kinases, CK1 and CK2 & {$[9]$} \\
\hline 1971 & Complete amino acid sequence of $a_{\text {s1 }}$ casein is determined & {$[15]$} \\
\hline 1972 & Casein kinase activity detected in Golgi fractions of lactating rat mammary gland & {$[12]$} \\
\hline $1988-89$ & "Genuine/Golgi" casein kinase (G-CK) consensus is different from those of CK2 and CK1; & {$[17,18]$} \\
\hline 1995 & Casein kinase-1 and casein kinase-2 renamed protein kinase CK1 and CK2 & {$[11]$} \\
\hline 1996 & $\begin{array}{l}\text { Specificity determinants for G-CK and the development of a synthetic peptide to detect G-CK activity with absolute } \\
\text { specificity }\end{array}$ & {$[16]$} \\
\hline 2012 & G-CK is identified as Fam20C & {$[2]$} \\
\hline
\end{tabular}




\begin{tabular}{|c|c|c|c|c|c|c|c|c|c|c|c|c|c|c|c|c|c|c|c|c|}
\hline & 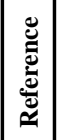 & $\overline{\widetilde{S}}$ & $\begin{array}{l}\bar{\infty} \\
\stackrel{\infty}{2}\end{array}$ & $\begin{array}{l}\square \\
\infty \\
\infty\end{array}$ & $\begin{array}{l}0 \\
0 \\
0\end{array}$ & $\underset{\infty}{\Gamma}$ & $\left|\begin{array}{l}\infty \\
\infty \\
\infty\end{array}\right|$ & $\begin{array}{l}\bar{\infty} \\
\stackrel{n}{n}\end{array}$ & $\underset{f}{\sigma}$ & \begin{tabular}{|l|}
$\mathbf{D}$ \\
$n$ \\
$n$
\end{tabular} & $\bar{\Phi}$ & $\mid$\begin{tabular}{|l}
$\infty$ \\
$\infty$ \\
$n$
\end{tabular} & $\sqrt{n}$ & $\sqrt{n}$ & $\bar{\Xi}$ & $\bar{\Xi}$ & 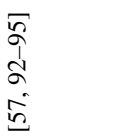 & $\begin{array}{l}\overline{2} \\
\dot{5} \\
i\end{array}$ & $\begin{array}{l}F \\
\sigma \\
\hat{i} \\
\dot{\tilde{n}}\end{array}$ & $\begin{array}{l}F \\
\text { F } \\
\tilde{\tilde{n}}\end{array}$ \\
\hline 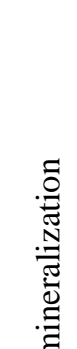 & 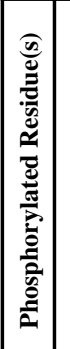 & $\begin{array}{l}5 \\
\text { 戛 } \\
\text { 离 } \\
0\end{array}$ & 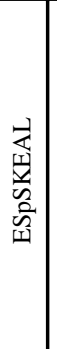 & 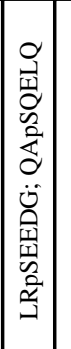 & 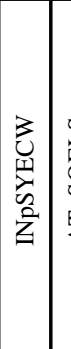 & 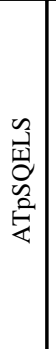 & 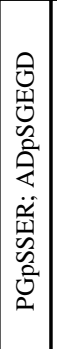 & 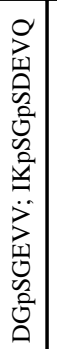 & 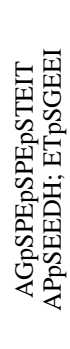 & 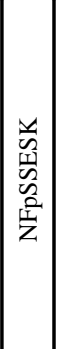 & 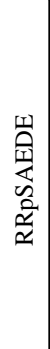 & 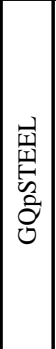 & 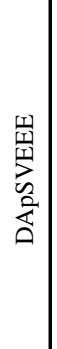 & 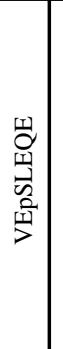 & $\begin{array}{l}\text { 前 } \\
\text { 恶 } \\
\text { 密 }\end{array}$ & 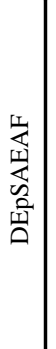 & 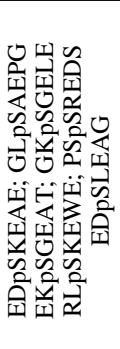 & 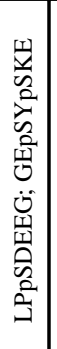 & 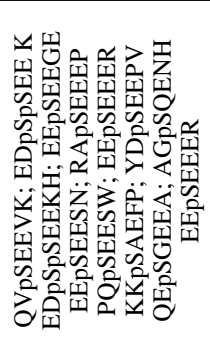 & 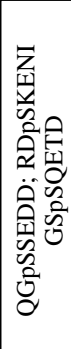 \\
\hline 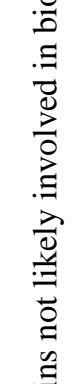 & 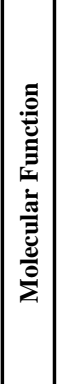 & 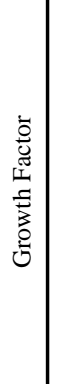 & 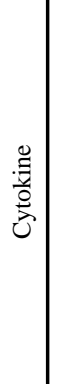 & 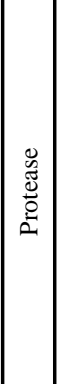 & 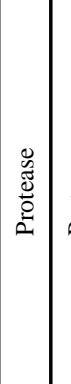 & 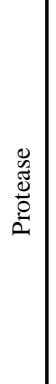 & 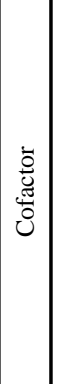 & 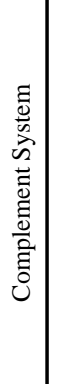 & 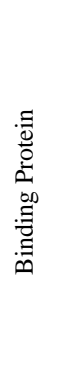 & 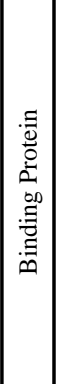 & 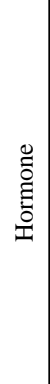 & 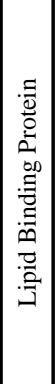 & 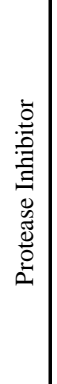 & 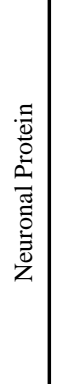 & 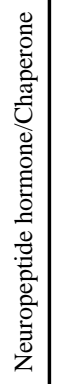 & 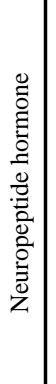 & 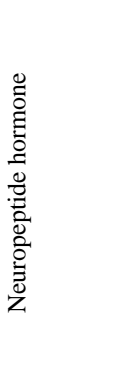 & 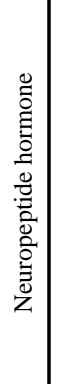 & 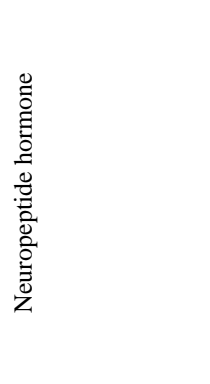 & 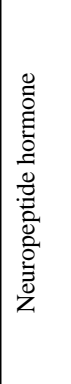 \\
\hline 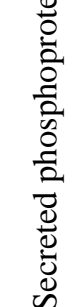 & 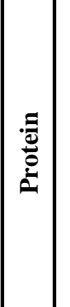 & $\sum_{n=}^{n}$ & 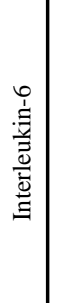 & $\mid \begin{array}{l}2 \\
\hat{v} \\
\tilde{v} \\
0\end{array}$ & 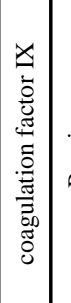 & $\begin{array}{l}\frac{1}{0} \\
\frac{2}{2} \\
2\end{array}$ & 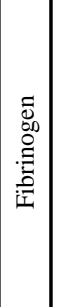 & 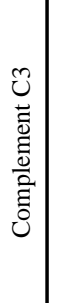 & $\begin{array}{l}\overline{\overrightarrow{0}} \\
\text { 酋 }\end{array}$ & 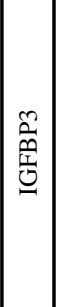 & 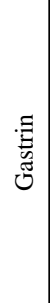 & \begin{tabular}{|l|} 
\\
0 \\
0 \\
0
\end{tabular} & 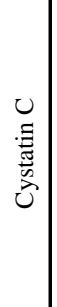 & 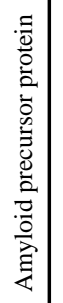 & 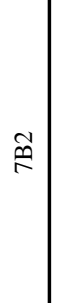 & 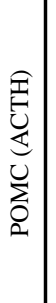 & 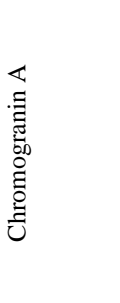 & 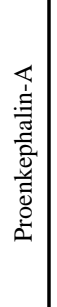 & 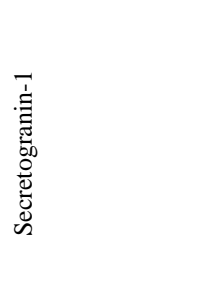 & 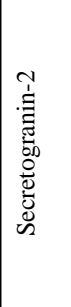 \\
\hline
\end{tabular}

